\title{
Negative shear lag effect of simply supported curved box girder
}

\author{
Hai Lin $\mathrm{Lu}^{1, \mathrm{a}}$, Zheng Tang ${ }^{1, \mathrm{~b}}$, Heng Cai, ${ }^{1, \mathrm{c}}$ and Xiao LongZhou ${ }^{1, \mathrm{~d}}$ \\ ${ }^{1}$ School of Resource and Civil Engineering, Wuhan Institute of Technology , Wuhan 430073, China; \\ b724873550@qq.com
}

Keywords: Curved box girder, negative shear lag effect, finite element, parametric analysis.

\begin{abstract}
In order to study the negative shear lag effect of simply supported curved box girder, spatial shell finite element models under different web width, box girder height and curvature radius are founded based on finite element principle, and parametric sensitivity analysis is carried out so as to reveal the distribution law of shear lag coefficients along full span direction. The results show that: (1) Along span direction, it presents negative shear lag effect at the top nodes of inside web and positive shear lag effect at the top nodes of outside web, there are critical points of positive and negative shear lag effect at cross section center.(2) Compared with straight girder, curved girder consider the influence of curvature, whose stress distribution of positive and negative shear lag are more non-uniform.(3) Positive and negative shear lag effect are greatly influenced by web width, box girder height and curvature radius; when the web width and box girder height increase, negative shear lag coefficients inside are smaller and smaller, while positive shear lag coefficients outside are growing, positive and negative shear lag effect both become more prominent, when the curvature radius is smaller, positive and negative shear lag effect inside and outside are more and more obvious.
\end{abstract}

\section{Introduction}

Curved box girder subjected to symmetrical loads, in its vertical bending plane, the normal stress caused by shear deformation is non-uniformed distributed along the flange width, the phenomenon, known as "shear lag effect [1]". According to the classical definition of shear lag, if the stress of the junction of rib is greater than the stress at cross section center, it is called "positive shear lag", otherwise, called"negative shear lag". In recent years, scholars home and abroad study a lot of positive shear lag, while the negative shear lag is less researched, based on box girder deflection analysis, Y.H.Zhang et al. [2] put forward a method to judge positive and negative shear lag of box girder, deducing discriminant expression by cross-section geometry and generalized moment; Y.F.Li et al [3] used the finite element theory to establish space finite element model, characteristics and the negative shear lag effect in main girder of cable-stayed bridge with single cable plane under the maximum negative moment affected by the live load forces are analyzed; Y.H.Zhang [4] studied negative shear lag factors of cantilever box girder with FEM, the results showed that the increasing of shear deformation, poisson ratio, width-height ratio and web thickness will intensify positive shear lag effect, the smaller width-span ratio and the larger stiffness ratio are, the more prominent positive and negative shear are. As you can see, the research above aim to study straight box girder, unlike straight box girder, spatial mechanical behavior of curved box girder is complicated, under symmetrical loads, there are not only coupling deformation of bending, torsion, and shear lag effect but also distortion and warping. A few research on negative shear lag effect have been carried out so far, most of which are limited to the straight box girder and cantilever curved box girder, while there are few research on simply supported curved box girder, therefore, it is very necessary to study it. In this paper, spatial shell finite element models under different web width, box girder height and curvature radius are founded based on finite element principle, and parametric sensitivity analysis is carried out so as to reveal the distribution law of shear lag coefficients along full span direction. 


\section{Curved box girder model}

Structure Size. Material and section size of box girder come from reference [5], an simply supported box girder model made of organic glass, Fig1 shows cross section size, structural parameters: $t_{1}=t_{2}=t_{3}=10 \mathrm{~mm}$, web width $b=200 \mathrm{~mm}$, the width of cantilever plate $b^{f}=100 \mathrm{~mm}$, the height of girder $h=100 \mathrm{~mm}$, curvature radius $r=2.5 \mathrm{~m}$, curvature angle $\theta=30^{\circ}$. material parameters: Elastic modulus $E=3000 \mathrm{MPa}$, Poisson's ratio $u=0.385$, density $\rho=1180 \mathrm{~kg} / \mathrm{m}^{3}$.

The Finite Element Model. 4-nodes shell63 element is adopted to establish the simply supported curved box girder model, there are 432 nodes and 416 elements in total, considering the constraint boundary conditions, four nodes at the junction between web and bottom flange are restricted, One end are $U_{x} 、 U_{y}, U_{z}$ and $U_{y}, U_{z}$, while $U_{x} 、 U_{y}$ and $U_{y}$ at the other end, finite element model is shown in Fig2.
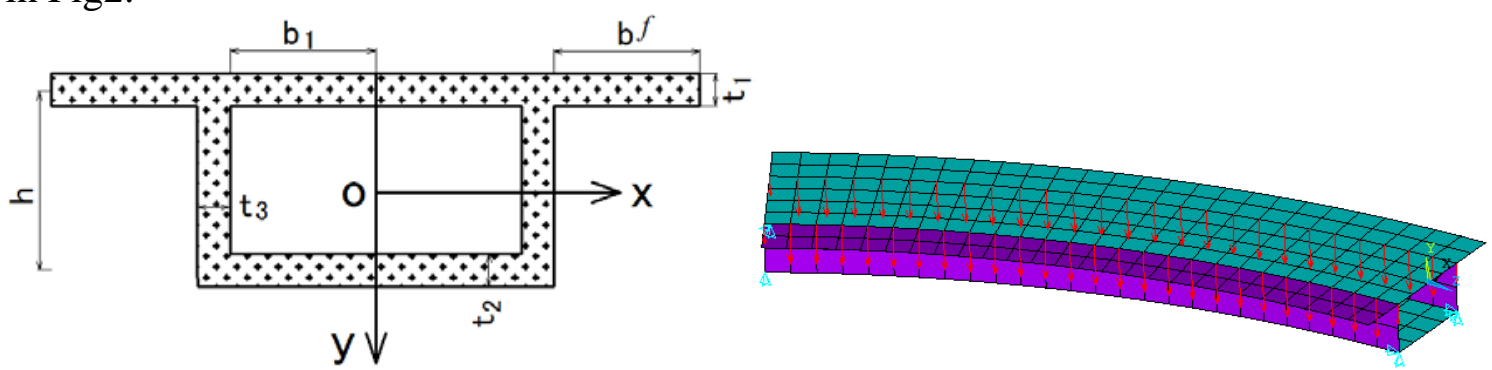

Fig.1 Cross section of box girder

Fig.2 Finite element model of curved box girder

Loading Conditions. This paper focuses on the negative shear lag effect of simply supported curved box girder,Uniform load $q=-100 \mathrm{~N} / \mathrm{m}$ is symmetrically applied in the junction between inner web, outer web and upper flange along full span direction. Load schema is shown in Fig3.

\section{Negative Shear Lag Effect}

The shear lag coefficient is defined as the [1].

$$
\lambda=\frac{\sigma}{\bar{\sigma}}
$$

Where $\lambda$ is the shear lag coefficient, $\sigma 、 \bar{\sigma}$ respectively, the stress value of the actual stress value and the calculated value based on elementary beam theory.

If $\lambda>1$, it is positive shear lag effect, while $\lambda<1$, it is negative shear lag effect. According to the finite element analysis results and based on equation (1), the shear lag coefficients distribution law along full span direction of curved box girder can be obtained. As is shown in Fig4, $\lambda^{n}$ and $\lambda^{w}$ respectively stand for shear lag coefficients at the top nodes of inside and outside web, $\lambda^{s}$ means the corresponding straight box girder shear lag coefficient at the top nodes of web.

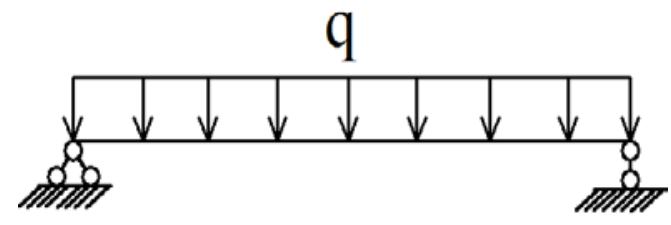

Fig.3 load schema

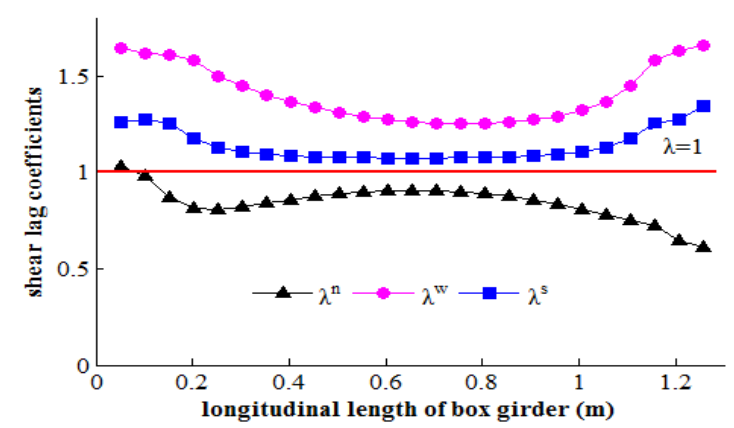

Fig.4 Distribution law of shear lag coefficients

It can be seen from Fig4:

(1) Under uniform loads, it presents obvious shear lag effect along full span direction of curved box girder, there is only negative shear lag at the top nodes of inside web, the minimum shear lag 
coefficient is 0.6 , while there is only positive shear lag at the top nodes of outside web, the maximum shear lag coefficient is1.6.

(2) Shear lag coefficient of straight box girder along the full span is greater than 1 , the shear lag effect is not obvious within a regional proximity to the mid-span, existing inertia areas of shear lag effect; shear lag coefficients of top nodes of outside web decreases firstly and then increases at mid-span along the longitudinal direction.

(3) Along the longitudinal direction, shear lag coefficient of top nodes of outsides web is larger than that of inside, shear lag coefficient of web top node of straight box girder is between them, which means curved girder consider the influence of curvature, whose stress distribution of positive and negative shear lag are more non-uniform.

\section{Parametric Analysis Of Shear Lag Effect}

In order to further study negative shear lag effect of curved box girder, in this paper, 3D spatial shell finite element model of simply supported curved box girder under different web width $(d=200$ 、 $300 、 400 \mathrm{~mm})$, box girder height $(h=100 、 150 、 200 \mathrm{~mm})$, curvature radius $(r=2.5 、 5 、 7.5 \mathrm{~m})$ are established, revealing the distribution law of shear lag coefficients along full span direction, the results are respectively shown in Fig.5 to Fig.7.

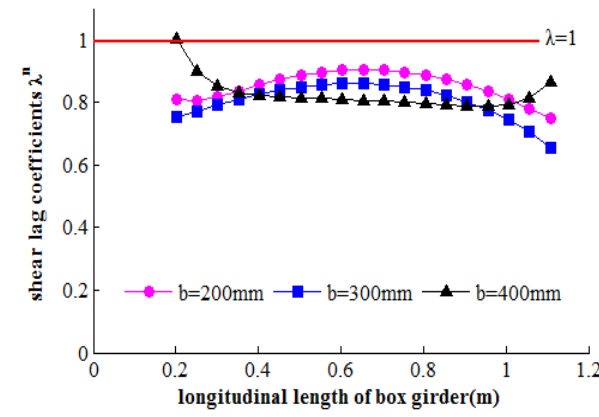

(a) inside

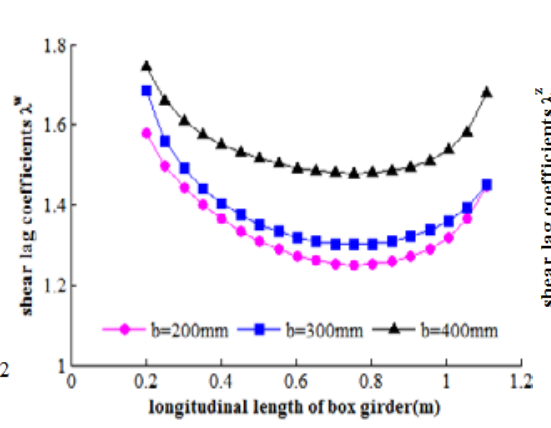

(b) outside

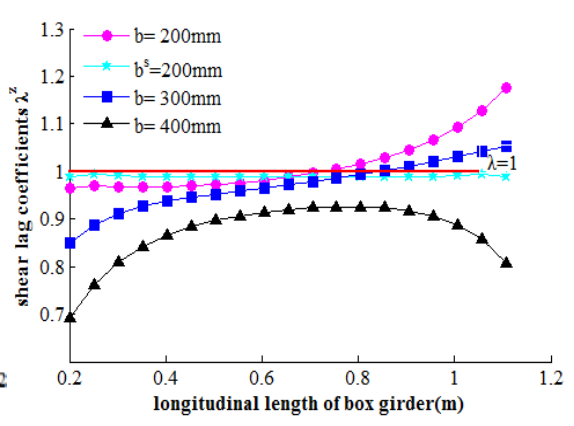

(c) cross section center

Fig.5 Influence of web width on shear lag coefficients

It can be seen from Fig.5:

(1) With the increasing of the web width, shear lag coefficients of top nodes of inside web become smaller and smaller from $2 / 13 \mathrm{~L}$ to $11 / 13 \mathrm{~L}$, the negative shear lag effect is more and more severe, when $b=200 、 300 \mathrm{~mm}$, shear lag coefficients increase firstly and then decrease, when $b=400 \mathrm{~mm}$, the situation is opposite, shear lag coefficients decreases firstly and then increases, the extreme value both appear at the mid span.

(2) For the top nodes of outside web, when web width increases, shear lag coefficients of the same node become bigger, which means shear lag effect becomes more and more severe; under three different web width, shear lag coefficients decrease firstly and then increase along the full span longitudinal direction, the extreme value also both appear at the mid span..

(3) At the cross section center, when $b=200 、 300 \mathrm{~mm}$, there are critical point of positive and negative shear lag, near the two supports, positive and negative shear lag effect is more prominent, when $b=400 \mathrm{~mm}$, there are only negative shear lag; shear lag coefficient of straight box girder is close to 1 and have no obvious change along full span direction.

As is shown in Fig.6:

(1) The top node of inside only have negative shear lag effect, with the increasing of box girder height, negative shear lag effect becomes more and more severe, along the full span of box girder, negative shear lag coefficient changes a little, and shear lag coefficients of three curved box girder heights fluctuate around $\mathbf{0 . 8}$. 


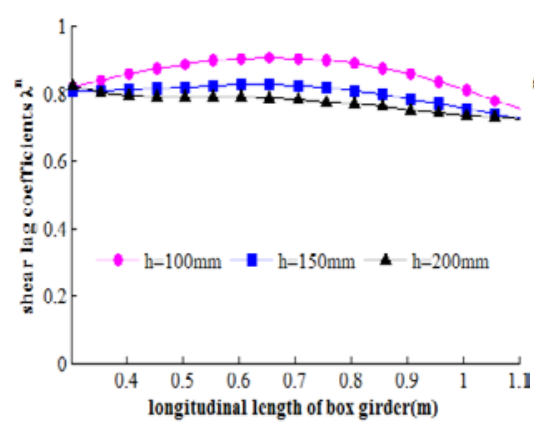

(a) inside

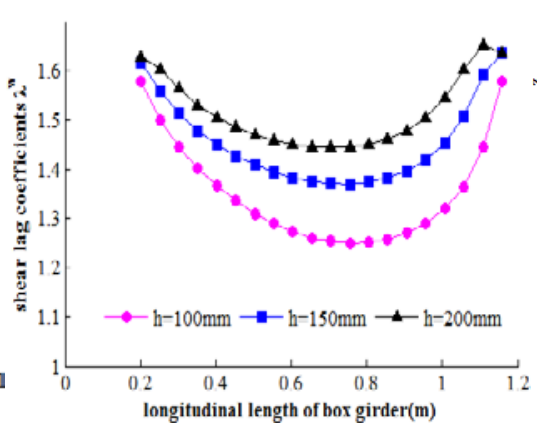

(b) outside

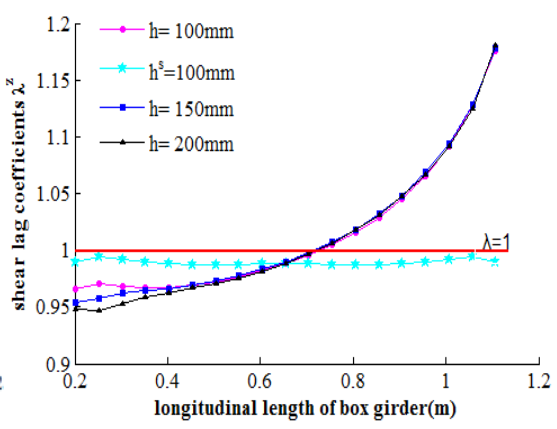

(c) cross section center

Fig.6 Influence of box girder height on shear lag coefficients

(2) For the top nodes of outside web, there are only positive shear lag effect, when curved box girder height increases, positive shear lag effect becomes more and more severe. Along full span direction, positive shear lag coefficient changes a lot, the extreme value appear at the mid span. Combine with (1), It indicates that reducing box girder height appropriately can weaken the positive and negative shear lag effect effectively.

(3) At the cross section center, under three different curved box girder heights, shear lag coefficients tend to boost, there are critical point of positive and negative shear lag at $L=0.7 \mathrm{~m}$. When curved box girder height increases, shear lag coefficient changes a little. Shear lag coefficients of top nodes of straight box girder are close to 1 along full span direction.

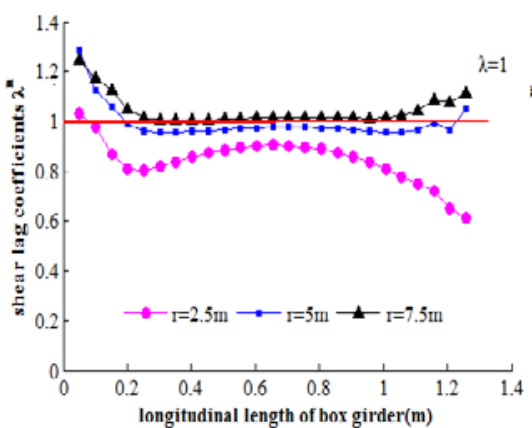

(a) inside

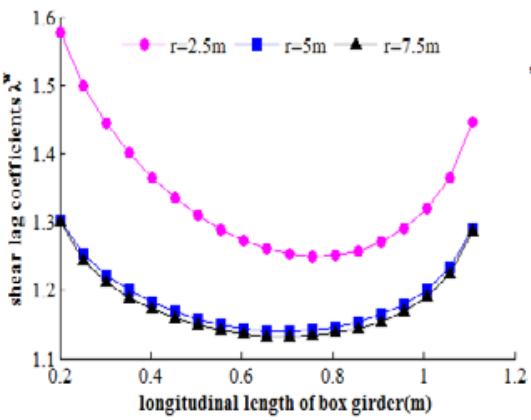

(b) outside

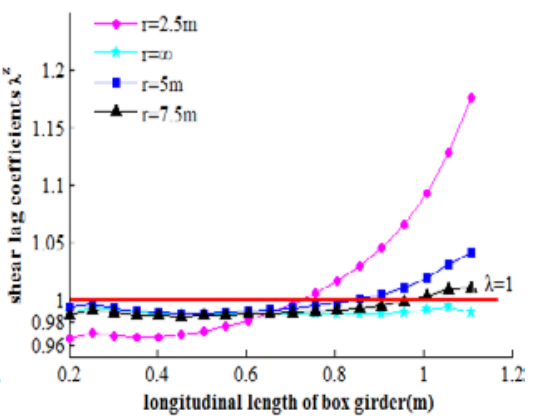

(c) cross section center

Fig.7 Influence of curvature radius on shear lag coefficients

Fig.7 indicates that:

(1) Under three different curvature radius, shear lag coefficients of top nodes of inside web shows great differences: when $r=2.5 \mathrm{~m}$, there are prominent negative shear lag effect. When curvature radius increases, negative shear lag effect are more and more not obvious. When $r=5,7.5 \mathrm{~m}$, there are a long inertia areas of shear lag effect

(2) For the top nodes of outside web, there are only positive shear lag effect. When $r=2.5 \mathrm{~m}$, shear lag effects are prominent. And when curvature radius increases, shear lag effect is more and more not obvious. When $r=5 、 7.5 \mathrm{~m}$, two curves of shear lag coefficients are almost the same.

(3) At the cross section center, when $r=2.5 \mathrm{~m}$, positive and negative shear lag effect are both severe. When curvature radius increases, shear lag effect are more and more not obvious. And its shear lag coefficients tends to that of straight box girder, which are close to 1 .

\section{Summary}

By analyzing spatial shell finite element model of single cell single room simply supported curved box girder, it can be concluded that:

(1)It shows a prominent shear lag effect along full span direction of curved box girder, there is only negative shear lag at the junction between inner web and upper flange, while there is only positive shear lag at the junction between outer web and upper flange, there are critical points of positive and negative shear lag effect at cross section center. 
(2)Along full span direction, shear lag coefficient at the junction between outer web and upper flange is greater than that of inside, and shear lag coefficient of straight box girder is between them. It shows that curved girder consider the influence of curvature, whose stress distribution of positive and negative shear lag are more non-uniform.

(3)Web width, height of box girder and radius of curvature have a great influence on both positive and negative shear lag effect.

Specifically:

1. When the web width increases, the negative shear lag coefficients inside get smaller and smaller and shear lag coefficients outside get bigger, positive and negative shear lag effect is more and more severe.

2. With the increasing of box girder height, the variation law of shear lag inside and outside is consistent with the web width. Therefore, reducing the height of the box girder appropriately can effectively weaken the positive and negative shear lag effect.

3. When curvature radius is smaller, the inner and outer positive and negative shear lag effect gets more prominent. In practical engineering, the large curvature box girder should be avoided.

\section{Acknowledgements}

This work was financially Supported by the National Natural Science Foundation of China (Project No. 51378404)and Innovation Fund of Wuhan Institute of Technology(Project No. CX2015042) these supports are gratefully acknowledged.

\section{References}

[1] H.F.Xiang, L.C.Fan, Advanced Theory of Bridge Structures,second ed.,China communication Press,Bei Jing, 2013.

[2] Y.H.Zhang, N.N.Li, Y.Liu. Discrimination between positive and negative shear lags in box girder by generalized moment, J. Journal of the China Railway Society. 33(9) (2011) 108-113.

[3] Y.F.Li, L.Liang, F.C.Wang. Research on negative shear lag effect in main girder of cable-stayed bridge with single cable plane under the live load forces,J. Chinese journal of applied mechanics. 30(2) (2013) 268-273.

[4] Y.H.Zhang. The experimental study and theoretical analysis in shear lag effect on thin-walled box-girder, D. Lanzhou: Lanzhou Jiaotong University. (2011) 45-51

[5] H.L.LU. Theoretical analysis and experimental study on shear-lag effect of prestress concrete curved box beam, D. Tianjin: Tianjin University (2005) 30-31. 